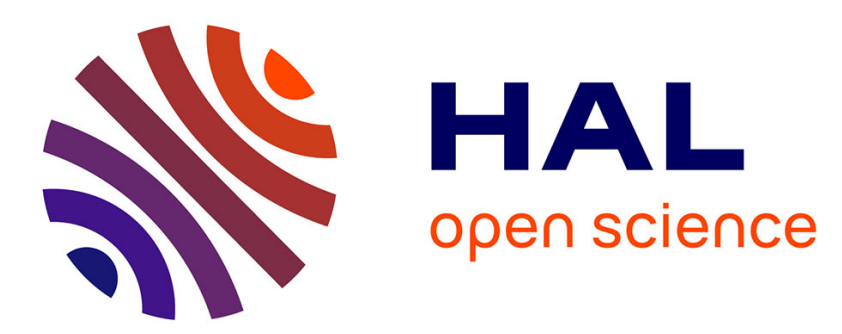

\title{
Parameterized Reduced Order Modeling of Misaligned Stacked Disks Rotor Assemblies
}

Vladislav Ganine, Denis Laxalde, Hannah Michalska, Christophe Pierre

\section{To cite this version:}

Vladislav Ganine, Denis Laxalde, Hannah Michalska, Christophe Pierre. Parameterized Reduced Order Modeling of Misaligned Stacked Disks Rotor Assemblies. Journal of Sound and Vibration, 2011, 330 (3), pp.445-460. 10.1016/j.jsv.2010.08.026 . hal-00511333

\section{HAL Id: hal-00511333 \\ https://hal.science/hal-00511333}

Submitted on 24 Aug 2010

HAL is a multi-disciplinary open access archive for the deposit and dissemination of scientific research documents, whether they are published or not. The documents may come from teaching and research institutions in France or abroad, or from public or private research centers.
L'archive ouverte pluridisciplinaire HAL, est destinée au dépôt et à la diffusion de documents scientifiques de niveau recherche, publiés ou non, émanant des établissements d'enseignement et de recherche français ou étrangers, des laboratoires publics ou privés. 


\title{
Parameterized Reduced Order Modeling of Misaligned Stacked Disks Rotor Assemblies
}

\author{
Vladislav Ganine ${ }^{\mathrm{a}} \quad$ Denis Laxalde $^{\mathrm{a}} \quad$ Hannah Michalska $^{\mathrm{b}}$ \\ Christophe Pierre ${ }^{\mathrm{a}}$ \\ ${ }^{a}$ Structural Dynamics and Vibration Laboratory \\ Department of Mechanical Engineering \\ b Department of Electrical and Computer Engineering \\ McGill University, Montreal, QC, Canada
}

\begin{abstract}
Light and flexible rotating parts of modern turbine engines operating at supercritical speeds necessitate application of more accurate but rather computationally expensive 3D FE modeling techniques. Stacked disks misalignment due to manufacturing variability in the geometry of individual components constitutes a particularly important aspect to be included in the analysis because of its impact on system dynamics. A new parametric model order reduction algorithm is presented to achieve this goal at affordable computational costs. It is shown that the disks misalignment leads to significant changes in nominal system properties that manifest themselves as additional blocks coupling neighboring spatial harmonics in Fourier space. Consequently, the misalignment effects can no longer be accurately modeled as equivalent forces applied to a nominal unperturbed system. The fact that the mode shapes become heavily distorted by extra harmonic content renders the nominal modal projection based methods inaccurate and thus numerically ineffective in the context of repeated analysis of multiple misalignment realizations. The significant numerical bottleneck is removed by employing an orthogonal projection onto the subspace spanned by first few Fourier harmonic basis vectors. The projected highly sparse systems are shown to accurately approximate the specific misalignment effects, to be inexpensive to solve using direct sparse methods and easy to parameterize with a small set of measurable eccentricity and tilt angle parameters. Selected numerical examples on an industrial scale model are presented to illustrate the accuracy and efficiency of the algorithm implementation.
\end{abstract}

Keywords: Rotordynamics ; model order reduction ; cyclic symmetry ; multi-stage ; misalignment ; parametric uncertainty

\section{Introduction}

Quantification of the effects of inevitable manufacturing variability is of growing importance to the design of engineering structures. In particular, flexible rotor-bearing systems 
show significant sensitivity of vibration behavior to variability in system properties. One important issue arising at turbine engine design and assembly stages is accurate prediction of the effects of stacked disks misalignment on rotor assembly flexural behavior.

Many industrial applications benefit from the classical rotordynamics analysis approaches established on one-dimensional beam type behavior hypothesis. The associated models have proved particularly effective means in many practical situations being relatively simple to build (although it may take considerable amount of manual effort to tune them [18]), leading to low order compact systems to solve. However, modern light-weight turbine engine designs feature rotating parts with complex geometrical configurations composed of flexible bladed disks elastically attached to thin-walled flexible shafts. Consequently, application of classical modeling techniques to such systems leads to disproportionally large modeling errors. The major shortcomings of 1D models are manifested in their failure to properly account for inertial coupling effects between bladed disks and shafts as well as for deformation and stress stiffening effects of shaft cross-sections $[12,4,11,5]$. Therefore for that class of problems more accurate but rather computationally expensive $2 \mathrm{D}$ or $3 \mathrm{D}$ rotordynamics techniques are unavoidable.

There have been several papers dealing with advanced modeling of rotor continuum. 2D Fourier axisymmetric FE formulation for rotordyanamics analysis has been studied in [23]. The theory involves some simplification of rotor geometry to a perfect axisymmetrical configuration. The paper by Jacquet-Richardet et al. [12], see also [3], describes a full 3D solid FE formulation to analyze spatially periodic systems (such as rotor assemblies with bladed disks). Practical application of it is somewhat limited by the assumption of same number of elementary sectors per rotor assembly, which can be worked around by employing the multi-stage cyclic symmetry coupling technique introduced in [15]. Spatial symmetry hypothesis of these techniques allows to reduce the size of the original problem by analyzing decoupled spatial harmonics separately.

For the problems of practical interest with deviations from symmetry the computational effort required to model the rotor continuum using 3D FE formulation is rather substantial. Both the large dimension of system and the large computational requirements render such models inadequate for repeated calculations necessary to examine all possible combinations of model parameters at various operating conditions. Moreover, model properties are often functions of rotational speed adding considerably to the degree of complexity. To facilitate the analysis, it is essential to have accurate low-order models that are significantly faster to solve than the original full model.

In fact, there is an extensive literature in the area of model order reduction methods applied to rotordynamics problems. Many of these reduction techniques are derived using a projection-based framework, in which the system variables and governing equations are projected onto low dimensional subspaces. These methods include either balanced truncation $[20,8,14]$ or projection onto modal subspace $[3,8,13,9,6]$. In the context of parameter-dependent systems analysis, the resulting model is of lower order, but it is not necessarily valid for all combinations of parameters. Many techniques developed for non-rotating structures analysis, for the most part straightforward extensions of the modal projection based algorithms, assume small parameter variation around a nominal 
point allowing to reuse the nominal modal basis vectors [2]. This approach is not always appropriate leading to significant approximation errors as is the case with large variations in geometry [10].

The main purpose of this paper is to present a new parameterized model order reduction technique to analyze the effects of variations in geometry caused by disk misalignment on rotor global flexural behavior. The nominal rotor with realistic geometry is discretized using 3D solid FE accounting for all rotational inertia effects in a body attached frame at selected rotation speeds. Nominal rotational periodicity enables us to manipulate FE meshes involving only one elementary sector per stage. The proposed algorithm, for clarity and emphasis, is based on the orthogonal projection of system equations onto a subspace spanned by a few Fourier basis vectors, namely corresponding to first three harmonics, which results in highly sparse systems. Most, if not all, of published work in the area $[7,17,1,22,19]$ models various deviations from a baseline model, termed "faults", (such as mass unbalance, rotor bow, misalignment, etc.) by representing them in terms of equivalent forces applied to a nominal system. In the proposed method the disks misalignment is introduced as perturbation to system matrices and corresponding centrifugal forces. One of the main results of this paper is to carry out the idea of using the discrete Fourier transform to decouple perfectly symmetrical systems (cyclic symmetry) one step further and to show that in fact, the subspace spanned by Fourier basis vectors can be efficiently reused to project certain type of geometrical perturbation terms owing to their special structure. It is shown that in so doing decent approximation properties can be achieved, while computational efficiency is retained, and above all, costly modal reanalysis for each misalignment realization can be avoided. Special attention has been given to parametrization of the ROM (Reduced Order Model). Disk misalignment "faults" are generated on-line as multiplicative perturbation terms, a function of small number of measurable eccentricity and tilt angle parameters, without resorting to FE software. Together with provided computational strategies it significantly simplifies and accelerates repeated ROM update and solution using modern direct sparse methods. The numerical examples illustrate harmonic contamination of low frequency modes brought about by the additional blocks coupling neighboring spatial harmonics in Fourier space. This leads to some insights that appear to be new as well as simple explanation of known physical phenomenæ, i.e., discussed in the literature effects of misalignment on the harmonic content of vibration response.

The article is organized as follows. In Section 2 the 3D rotordynamics formulation for systems featuring cyclic symmetry is reviewed and the basic notions used in the construction of our computational technique are introduced. Section 3 contains the numerical examples examining the effects of disk misalignment on system response, confirming the accuracy of the proposed algorithm and presenting a statistical analysis case. Concluding remarks are given in Section 4. 


\section{Theoretical background}

\section{$2.13 \mathrm{D}$ rotordynamics equations of motion}

Consider a FE model of a lightly damped rotating structure. The equations of motion in the body fixed coordinate frame assuming constant angular velocity can be expressed as $[12,11]$

$$
\begin{gathered}
{\left[\mathbf{K}_{E}+\mathbf{K}_{G}\left(\mathbf{u}_{S}\right)-\mathbf{K}_{C}\left(\Omega^{2}\right)\right] \mathbf{u}_{S}=\mathbf{F}_{C}\left(\Omega^{2}\right)} \\
\mathbf{M} \ddot{\mathbf{u}}_{D}+\mathbf{C}(\Omega) \dot{\mathbf{u}}_{D}+\left[\mathbf{K}_{E}+\mathbf{K}_{G}\left(\mathbf{u}_{S}\right)-\mathbf{K}_{C}\left(\Omega^{2}\right)\right] \mathbf{u}_{D}=\mathbf{F}
\end{gathered}
$$

where $\mathbf{M} \in \mathbb{R}^{n \times n}$ is a symmetric positive definite mass matrix, $\mathbf{K}_{E} \in \mathbb{R}^{n \times n}$ is a symmetric semi-definite elastic stiffness matrix. Two rotational aspects are taken into account. The Coriolis forces proportional to the velocities are introduced through a skew-symmetric positive semi-definite pseudo-damping matrix $\mathbf{C} \in \mathbb{R}^{n \times n}$. The centrifugal forces generate spin softening matrix $\mathbf{K}_{C}\left(\Omega^{2}\right) \in \mathbb{R}^{n \times n}$ and a geometric stiffness matrix $\mathbf{K}_{G}\left(\mathbf{u}_{S}\right) \in \mathbb{R}^{n \times n}$ representing stress stiffening effects. $\quad \mathbf{F}_{C} \in \mathbb{R}^{n}$ denotes the nodal centrifugal forcing vector, $\Omega$ is the rotational speed. $\mathbf{u}_{S} \in \mathbb{R}^{n}$ is the static equilibrium position of the structure under centrifugal loading found by solving nonlinear equation (1), $\mathbf{u}_{D} \in \mathbb{R}^{n}$ is the small dynamic displacement around the static equilibrium and $\mathbf{F} \in \mathbb{R}^{n}$ is a harmonic excitation vector. Note the dependence of system matrices on rotor speed $\Omega$.

Nominal bladed disks-shaft assemblies at each individual stage feature rotational periodicity that in a cylindrical coordinate frame implies the block-circulant structure of system matrices [25]. Using that property, we can decouple both static (1) and dynamic (2) equations at an individual stage level into $N / 2$ smaller problems by applying discrete Fourier transform

$$
\left(\mathbf{W}^{*} \otimes \boldsymbol{I}\right)\left[\mathbf{K}_{E}+\mathbf{K}_{G}\left(\mathbf{u}_{S}\right)-\mathbf{K}_{C}\left(\Omega^{2}\right)\right](\mathbf{W} \otimes \boldsymbol{I})\left(\mathbf{W}^{*} \otimes \boldsymbol{I}\right) \mathbf{u}_{S}=\left(\mathbf{W}^{*} \otimes \boldsymbol{I}\right) \mathbf{F}_{C}\left(\Omega^{2}\right)
$$

$$
\begin{aligned}
\left(\mathbf{W}^{*} \otimes \boldsymbol{I}\right) \mathbf{M}(\mathbf{W} \otimes \boldsymbol{I})\left(\mathbf{W}^{*} \otimes \boldsymbol{I}\right) \ddot{\mathbf{u}}_{D}+\left(\mathbf{W}^{*} \otimes \boldsymbol{I}\right) \mathbf{C}(\Omega)(\mathbf{W} \otimes \boldsymbol{I})\left(\mathbf{W}^{*} \otimes \boldsymbol{I}\right) \dot{\mathbf{u}}_{D}+ \\
\left(\mathbf{W}^{*} \otimes \boldsymbol{I}\right)\left[\mathbf{K}_{E}+\mathbf{K}_{G}\left(\mathbf{u}_{S}\right)-\mathbf{K}_{C}\left(\Omega^{2}\right)\right](\mathbf{W} \otimes \boldsymbol{I})\left(\mathbf{W}^{*} \otimes \boldsymbol{I}\right) \mathbf{u}_{D}=\left(\mathbf{W}^{*} \otimes \boldsymbol{I}\right) \mathbf{F}
\end{aligned}
$$

where the discrete Fourier transform expressed in matrix form is defined as

$$
\mathbf{W}=\frac{1}{\sqrt{N}}\left[\begin{array}{cccc}
1 & 1 & \cdots & 1 \\
1 & e^{-\mathrm{j} \frac{2 \pi}{N}} & \cdots & e^{-\mathrm{j} \frac{2(N-1) \pi}{N}} \\
1 & e^{-\mathrm{j} \frac{4 \pi}{N}} & \cdots & e^{-\mathrm{j} \frac{4(N-1) \pi}{N}} \\
\vdots & \vdots & \ddots & \vdots \\
1 & e^{-\mathrm{j} \frac{2(N-1) \pi}{N}} & \cdots & e^{-\mathrm{j} \frac{2(N-1)(N-1) \pi}{N}}
\end{array}\right]
$$

here $N$ denotes the number of elementary sectors. The system equations of motion then become

$$
\left[\widetilde{\mathbf{K}}_{h E}+\widetilde{\mathbf{K}}_{h G}\left(\tilde{\mathbf{u}}_{h S}\right)-\widetilde{\mathbf{K}}_{h C}\left(\Omega^{2}\right)\right] \tilde{\mathbf{u}}_{h S}=\tilde{\mathbf{F}}_{h C}\left(\Omega^{2}\right)
$$




$$
\widetilde{\mathbf{M}}_{h} \ddot{\tilde{\mathbf{u}}}_{h D}+\widetilde{\mathbf{C}}_{h}(\Omega) \dot{\tilde{\mathbf{u}}}_{h D}+\left[\widetilde{\mathbf{K}}_{h E}+\widetilde{\mathbf{K}}_{h G}\left(\tilde{\mathbf{u}}_{h S}\right)-\widetilde{\mathbf{K}}_{h C}\left(\Omega^{2}\right)\right] \tilde{\mathbf{u}}_{h D}=\tilde{\mathbf{F}}_{h}
$$

where subscript $h$ describes the association to a Fourier harmonic. In most practical situations one can strongly reduce the order of the problem by selecting first harmonics $h=0$ and $h=1$ ( $h=0$ corresponds to torsion and longitudinal displacements, $h=1$ to bending). Truncated higher order harmonics are mainly responsible for disk dominated motion or for deformation of shaft cross section having little to no effect on the global bending behavior of the rotor assembly [11].

\subsection{Modeling of disk misalignment}

Consider a multi-stage system of $S$ cyclic symmetrical structures sharing the same axis of rotational symmetry. As it is known, the result of changes due to manufacturing imperfections in the geometry on inter-stage interfaces may result in static or dynamic imbalances in rotor plus non-isotropic variations of stiffness [21]. Assuming that other imperfections of each stage are negligible, i.e. they preserve rotational symmetry, we can describe these effects by orientation and position of an individual stage given by an arbitrary rotation in terms of two Euler angles $\theta_{x}, \theta_{y}$ and two offsets $\Delta x, \Delta y$, as shown in Fig. 1.

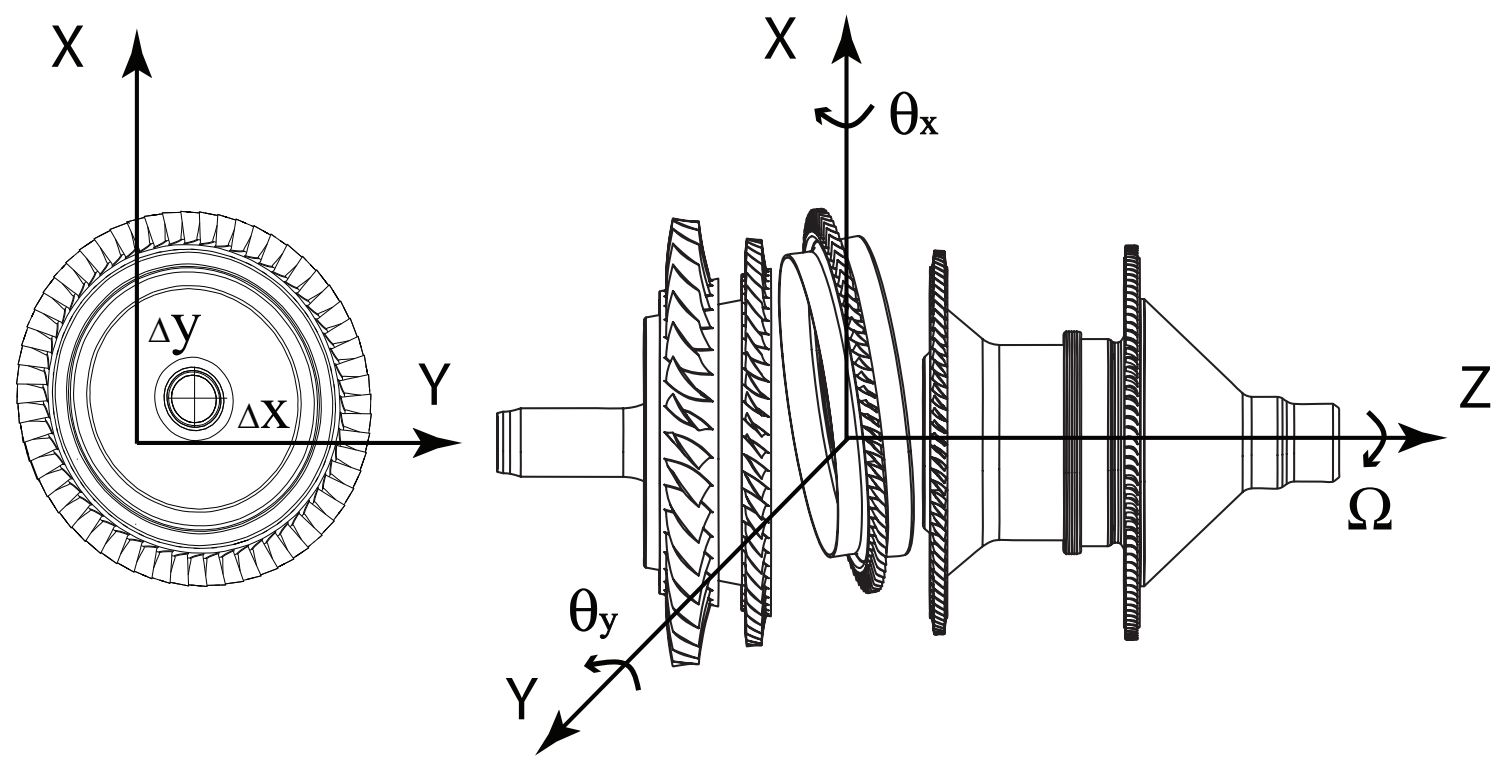

Figure 1: Stacked disks assembly misalignment (exaggerated) expressed in terms of two Euler angles $\theta_{x}, \theta_{y}$ and two offsets $\Delta x, \Delta y$.

Thus the perturbed position of a node that belong to $n_{t h}$ sector of $s_{t h}$ stage in cylindrical coordinate system in which the Z-axis is coincident with the shaft axis of 
rotation can be computed in three steps as follows

$$
\begin{aligned}
& \left\{\begin{array}{l}
x \\
y \\
z
\end{array}\right\}=\mathbf{R}_{z}\left\{\begin{array}{l}
r \\
\theta \\
z
\end{array}\right\} \\
& \left\{\begin{array}{l}
x^{\prime} \\
y^{\prime} \\
z^{\prime}
\end{array}\right\}=\mathbf{R}_{y} \mathbf{R}_{x}\left\{\begin{array}{c}
x+\Delta x \\
y+\Delta y \\
z
\end{array}\right\} \\
& \left\{\begin{array}{l}
r^{\prime} \\
\theta^{\prime} \\
z^{\prime}
\end{array}\right\}=\mathbf{R}_{z}^{T} \mathbf{R}_{z}^{\prime T}\left\{\begin{array}{l}
x^{\prime} \\
y^{\prime} \\
z^{\prime}
\end{array}\right\}
\end{aligned}
$$

where the rotation matrices

$$
\begin{gathered}
\mathbf{R}_{x}=\left[\begin{array}{ccc}
1 & 0 & 0 \\
0 & \cos \theta_{x} & -\sin \theta_{x} \\
0 & \sin \theta_{x} & \cos \theta_{x}
\end{array}\right], \mathbf{R}_{y}=\left[\begin{array}{ccc}
\cos \theta_{y} & 0 & \sin \theta_{y} \\
0 & 1 & 0 \\
-\sin \theta_{y} & 0 & \cos \theta_{y}
\end{array}\right] \\
\mathbf{R}_{z}=\left[\begin{array}{ccc}
\cos \alpha & -\sin \alpha & 0 \\
\sin \alpha & \cos \alpha & 0 \\
0 & 0 & 1
\end{array}\right], \mathbf{R}_{z}^{\prime}=\left[\begin{array}{ccc}
\cos \Delta \theta_{z} & -\sin \Delta \theta_{z} & 0 \\
\sin \Delta \theta_{z} & \cos \Delta \theta_{z} & 0 \\
0 & 0 & 1
\end{array}\right]
\end{gathered}
$$

$\alpha=2(n-1) \pi / N$ denotes $n_{t h}$ elementary sector rotation angle about the axis of symmetry and $\Delta \theta_{z}$ is the perturbation of that angle due to rotations and translations in Cartesian coordinate frame.

$$
\begin{array}{ll}
\Delta \theta_{z}=\arccos \left(x^{\prime} / r^{\prime}\right)-\arccos (x / r) & \text { if } y^{\prime} \geq 0 \\
\Delta \theta_{z}=-\left(\arccos \left(x^{\prime} / r^{\prime}\right)-\arccos (x / r)\right) & \text { if } y^{\prime}<0
\end{array}
$$

Using the definitions above we can write the equations motion of a misaligned stage in cylindrical coordinate frame

$$
\begin{gathered}
{\left[\mathbf{P}^{T}\left(\mathbf{K}_{E}-\mathbf{K}_{C}\left(\Omega^{2}\right)\right) \mathbf{P}+\widehat{\mathbf{K}}_{G}\left(\widehat{\mathbf{u}}_{S}\right)\right] \widehat{\mathbf{u}}_{S}=\widehat{\mathbf{F}}_{C}\left(\Omega^{2}\right)} \\
\mathbf{P}^{T} \mathbf{M} \mathbf{P} \ddot{\hat{\mathbf{u}}}_{D}+\mathbf{P}^{T} \mathbf{C}(\Omega) \mathbf{P} \dot{\hat{\mathbf{u}}}_{D}+\left[\mathbf{P}^{T}\left(\mathbf{K}_{E}-\mathbf{K}_{C}\left(\Omega^{2}\right)\right) \mathbf{P}+\widehat{\mathbf{K}}_{G}\left(\widehat{\mathbf{u}}_{S}\right)\right] \widehat{\mathbf{u}}_{D}=\mathbf{F}
\end{gathered}
$$

here $\mathbf{P}=\underset{i=1, \ldots, n_{\text {nodes }} N}{\text { Bdiag }}\left\{\mathbf{R}_{z} \mathbf{R}_{z}^{\prime} \mathbf{R}_{y x}^{T} \mathbf{R}_{z}^{T}\right\}$, and $n_{\text {nodes }}$ denotes the number of nodes in an elementary sector.

Several basic simplifying assumptions can be taken to reduce computational effort without compromising the accuracy. As pointed out in [16], the form of the mass matrix of $3 D$ solid finite elements does not change under orthogonal coordinate transformation, i.e. $\mathbf{P}^{T} \mathbf{M P}=\mathbf{M}$. The cost of $\mathrm{FE}$ reevaluation of the nodal centrifugal force for each misalignment scenario can be avoided by employing the lumped mass formulation, i.e. the $i_{t h}$ nodal centrifugal force can be expressed in the form

$$
\widehat{\mathbf{f}}_{i C}\left(\Omega^{2}\right) \approx \Omega^{2} \mu_{i} r_{i}^{\prime}
$$


where $\mu_{i}$ is the lumped mass entry, $r_{i}^{\prime}$ is perturbed radial coordinate in the cylindrical frame.

Because the deformations due to centrifugal forcing are assumed to be small in this problem due to low rotational speeds $\Omega$ and low magnitude of uncertain parameters $\left(\theta_{x}, \theta_{y}, \Delta x, \Delta y\right)$, the perturbed centrifugal stiffening matrix can be reasonably approximated as the elastic stiffness matrix, i.e. $\left.\widehat{\mathbf{K}}_{G}\left(\widehat{\mathbf{u}}_{S}\right)=\mathbf{P}^{T} \mathbf{K}_{G}\left(\mathbf{u}_{S}\right)\right) \mathbf{P}$. The validity of that assumption will be verified numerically on the industrial scale model later in the paper.

Employing these simplifications, the equations of motion of the misaligned stage take form

$$
\begin{gathered}
\mathbf{P}^{T}\left(\mathbf{K}_{E}+\mathbf{K}_{G}\left(\mathbf{u}_{S}\right)-\mathbf{K}_{C}\left(\Omega^{2}\right)\right) \mathbf{P} \widehat{\mathbf{u}}_{S}=\widehat{\mathbf{F}}_{C}\left(\Omega^{2}\right) \\
\mathbf{M} \ddot{\hat{\mathbf{u}}}_{D}+\mathbf{P}^{T} \mathbf{C}(\Omega) \mathbf{P} \dot{\widehat{\mathbf{u}}}_{D}+\mathbf{P}^{T}\left(\mathbf{K}_{E}+\mathbf{K}_{G}\left(\mathbf{u}_{S}\right)-\mathbf{K}_{C}\left(\Omega^{2}\right)\right) \mathbf{P} \widehat{\mathbf{u}}_{D}=\mathbf{F}
\end{gathered}
$$

Eq. (11) can be interpreted simply as follows: the effect of misalignment is introduced by a multiplicative perturbation to stiffness matrix accounting for its non-isotropic variations, plus centrifugal forcing that includes mass imbalance forces.

\subsection{Misalignment representation in Fourier domain}

The preceding discussion was primarily concerned with modeling of misalignment in cylindrical coordinate system. For reduced order model formulation we explore the Fourier domain representation of the multiplicative perturbation matrix $\mathbf{P}$. Let the nominal elastic plus geometric stiffness matrices be partitioned into $H$ harmonic blocks, then we can introduce the misalignment as follows

$$
\widehat{\widetilde{\mathbf{K}}}=(\mathbf{W} \otimes \boldsymbol{I}) \mathbf{P}^{T}\left(\mathbf{W}^{*} \otimes \boldsymbol{I}\right) \underset{h=0, \ldots, H}{\widetilde{B}} \operatorname{diag}\left\{\widetilde{\mathbf{K}}_{h E}+\widetilde{\mathbf{K}}_{h G}-\widetilde{\mathbf{K}}_{h C}\right\}(\mathbf{W} \otimes \boldsymbol{I}) \mathbf{P}\left(\mathbf{W}^{*} \otimes \boldsymbol{I}\right)
$$

The Discrete Fourier Transform (DFT) of each component of $\mathbf{P}$ can be evaluated separately, moreover one may consider the multiplication $\widetilde{\mathbf{P}}=(\mathbf{W} \otimes \boldsymbol{I}) \mathbf{P}\left(\mathbf{W}^{*} \otimes \boldsymbol{I}\right)$ as application of the transform $n_{d o f} n_{d o f}$ times to $N \times N$ matrices, where $n_{d o f}$ is the number of degrees of freedom of an elementary sector, $N$ is number of sectors. Clearly, the rotation matrices $\mathbf{R}_{x}$ and $\mathbf{R}_{y}$ constant for all sectors are invariant under transform $(\mathbf{W} \otimes I) \mathbf{R}_{y} \mathbf{R}_{x}\left(\mathbf{W}^{*} \otimes I\right)=\mathbf{R}_{y} \mathbf{R}_{x}$

It can be shown that a DFT of a harmonic train that generate cosine and sine entries of the rotational matrices $\mathbf{R}_{z}$ and $\mathbf{R}_{z}^{\prime}$ are of the form [24]

$$
\begin{aligned}
\cos \left(\frac{2 \pi n r}{N}\right) \delta(|n-m|) \Leftrightarrow & \frac{1}{2}(\delta(|u-v|-r)+\delta(|u-v|-(N-r))) \\
\sin \left(\frac{2 \pi n r}{N}\right) \delta(|n-m|) \Leftrightarrow & \frac{1}{2}(-j \delta(u-v-r)-j \delta(u-v-(N-r))+ \\
& +j \delta(v-u-r)+j \delta(v-u-(N-r)))
\end{aligned}
$$

where $\delta$ is the Dirac delta function, $n, m, u, v=1, \ldots, N$ are row and column indices of an $N \times N$ block and its transform correspondingly. The details of derivation are given 
in the Appendix. For rotational matrix $\mathbf{R}_{z}$, where frequency of the harmonic periodic train is $r=1$, this leads to tri-diagonal matrices of the form

$$
\begin{array}{r}
\widetilde{\mathbf{R}}_{\cos \alpha}=\left(\begin{array}{cccccc}
0 & 0.5 & & & & 0.5 \\
0.5 & \ddots & \ddots & & & \\
& \ddots & \ddots & \ddots & & \\
& & \ddots & \ddots & \ddots & \\
& & & \ddots & \ddots & 0.5 \\
0.5 & & & & 0.5 & 0
\end{array}\right) \\
\widetilde{\mathbf{R}}_{\sin \alpha}=\left(\begin{array}{cccccc}
0 & -\mathrm{j} 0.5 & & & & \mathrm{j} 0.5 \\
\mathrm{j} 0.5 & \ddots & \ddots & & & \\
& \ddots & \ddots & \ddots & & \\
& & \ddots & \ddots & \ddots & \\
-\mathrm{j} 0.5 & & & & \mathrm{j} 0.5 & 0
\end{array}\right)
\end{array}
$$

Note that for real conjugate-even sequences with real-valued DFT employed the entries 0.5 and $\pm \mathrm{j} 0.5$ at $\delta(|u-v|-(N-r))$ locations disappear. It follows readily that the transform $(\mathbf{W} \otimes \boldsymbol{I}) \mathbf{R}_{z}\left(\mathbf{W}^{*} \otimes \boldsymbol{I}\right)$ need not be evaluated numerically.

The non-zero structure of the transform of matrix $\mathbf{R}_{z}^{\prime}$ is again banded due to nearly harmonic periodic nature of $\Delta \theta_{z}$ with frequency $r=1$. Therefore the transformed entries $\cos \Delta \theta_{z}$ will be dominated by double frequency $r=2$ components, while $\sin \Delta \theta_{z}$ entries will retain the same $r=1$ frequency. Observe, that the exact values of the transformed matrices will be dependent on the phase of the periodic train as a function of misalignment parameters $\left(\theta_{x}, \theta_{y}, \Delta x, \Delta y\right)$ and thus have to be computed.

With the assumption of small in norm perturbation the product of the nominal blockdiagonal stiffness matrix with Fourier domain representation of the misalignment matrix given in Eq. (13) will essentially result in a block-banded matrix

$$
\widehat{\widetilde{\mathbf{K}}}=\left(\begin{array}{cccccc}
B_{0} & B_{0,1} & B_{0,2} & & & \\
B_{0,1}^{T} & \ddots & \ddots & \ddots & & \\
B_{0,2}^{T} & \ddots & \ddots & \ddots & \ddots & \\
& \ddots & \ddots & \ddots & \ddots & B_{H-2, H} \\
& & \ddots & \ddots & \ddots & B_{H-1, H} \\
& & & B_{H-2, H}^{T} & B_{H-1, H}^{T} & B_{H}
\end{array}\right)
$$

where $2 n_{d o f} \times 2 n_{d o f}$ submatrix $B_{h}$ is an original perturbed harmonic block and $B_{h, h+1}$ is a $2 n_{d o f} \times 2 n_{\text {dof }}$ term introduced by pre- and post-multiplication that couples neighboring harmonics, such that $\left\|B_{h, h+1}\right\| \ll\left\|B_{h}\right\|$ for small variations of parameters $\left(\theta_{x}, \theta_{y}, \Delta x, \Delta y\right)$. 
Then other coupling off-diagonal blocks of higher order are negligible in norm $\left\|B_{h, h+2}\right\| \ll$ $\left\|B_{h, h+1}\right\|$. In particular, that result implies that one may obtain a reasonable accuracy reduced order system to analyze harmonic one behavior through a simple truncation by retaining first three harmonic blocks with respective coupling terms.

\subsection{Interstage coupling and assembly}

Employing small magnitude perturbation assumption that does not significantly change interstage interface the connection of misaligned adjacent stages can be achieved through multi-stage cyclic symmetry coupling. Consider the displacements compatibility condition between adjacent stages $s$ and $s+1$ defined in physical coordinates by

$$
\mathbf{A} \mathbf{u}_{b}^{s}-\mathbf{u}_{b}^{s+1}=0
$$

in which $\mathbf{A}$ is a Boolean connectivity matrix which makes the two interstage meshes compatible. The multi-stage cyclic symmetry coupling procedure defines independent compatibility relations between compatible (in terms of harmonic index) cyclic components of adjacent stages. Accordingly, given a cyclic harmonic of the rotor $n$, Eq. (15) is rewritten using cyclic harmonics $p_{s}(n)$ and $p_{s+1}(n)$ defined according to aliasing of respective Fourier bases of stage $s$ and $s+1$ :

$$
\left(\mathbf{w}_{p_{s+1}(n)}^{s+1}{ }^{*} \otimes \mathbf{I}\right) \mathbf{A}\left(\mathbf{w}_{p_{s}(n)}^{s} \otimes \mathbf{I}\right) \tilde{\mathbf{u}}_{b, p_{s}(n)}^{s}-\tilde{\mathbf{u}}_{b, p_{s+1}(n)}^{s+1}=0
$$

$\mathbf{w}_{p_{s}(n)}^{s}$ a column of Fourier transform matrix associated with harmonic $p_{s}(n) \in\left[0, N_{s}\right]$ of stage $s$, subscript $b$ denotes degrees-of-freedom on the inter-stage boundary. For further details, please refer to [15]. In order to eliminate the duplicated cyclic components of the interstage boundary of stage $s+1$ a rectangular coupling matrix is defined as

$$
\mathcal{T}=\left[\begin{array}{cccc}
\boldsymbol{I}_{b, s} & \mathbf{0} & \mathbf{0} & \cdots \\
\mathbf{0} & \boldsymbol{I}_{i, s} & \mathbf{0} & \cdots \\
\mathcal{B}_{p} & \mathbf{0} & \mathbf{0} & \ldots \\
\mathbf{0} & \mathbf{0} & \boldsymbol{I}_{i, s+1} & \cdots \\
\vdots & \vdots & \vdots & \ddots
\end{array}\right]
$$

where

$$
\mathcal{B}_{p}=\left(\mathbf{w}_{p_{s+1}(n)}^{s+1}{ }^{*} \otimes \mathbf{I}\right) \mathbf{A}\left(\mathbf{w}_{p_{s}(n)}^{s} \otimes \mathbf{I}\right)
$$

It follows that the equations of motion of the coupled misaligned rotor system, for $h=0, \ldots, 2$ can be expressed as

$$
\begin{gathered}
\mathcal{T}^{T} \widetilde{\mathbf{P}}^{T}\left[\widetilde{\mathbf{K}}_{h E}+\widetilde{\mathbf{K}}_{h G}-\widetilde{\mathbf{K}}_{h C}\right] \widetilde{\mathbf{P}} \mathcal{T} \widehat{\tilde{\mathbf{u}}}_{h S}=\widehat{\tilde{\mathbf{F}}}_{h C} \\
\mathcal{T}^{T} \widetilde{\mathbf{M}}_{h} \mathcal{T} \ddot{\tilde{\mathbf{u}}}_{h D}+\mathcal{T}^{T} \widetilde{\mathbf{P}}^{T} \widetilde{\mathbf{C}}_{h} \widetilde{\mathbf{P}} \mathcal{T} \dot{\tilde{\mathbf{u}}}_{h D}+\mathcal{T}^{T} \widetilde{\mathbf{P}}^{T}\left[\widetilde{\mathbf{K}}_{h E}+\widetilde{\mathbf{K}}_{h G}-\widetilde{\mathbf{K}}_{h C}\right] \widetilde{\mathbf{P}} \mathcal{T} \widehat{\tilde{\mathbf{u}}}_{h D}=\tilde{\mathbf{F}}_{h}
\end{gathered}
$$




\subsection{Algorithm for repeated ROM evaluation}

The main important result of the proposed method is that the complexity of solving Eqs. (18) and (19) can be greatly reduced if the linear systems are solved sequentially instead of calculating the matrix product, a highly populated matrix, and then solving it. However, typically the nominal uncoupled system is severely ill-conditioned due to the presence of rigid body modes. To improve the conditioning of nominal uncoupled system we propose to take advantage of the orthogonality properties of Fourier coefficients. If we define a rectangular coupling matrix

$$
\mathcal{T}_{0.5}=\mathcal{T}\left[\begin{array}{cccc}
0.5 \boldsymbol{I}_{b, s} & \mathbf{0} & \mathbf{0} & \cdots \\
\mathbf{0} & \boldsymbol{I}_{i, s} & \mathbf{0} & \cdots \\
\mathbf{0} & \mathbf{0} & \mathbf{0} & \cdots \\
\mathbf{0} & \mathbf{0} & \boldsymbol{I}_{i, s+1} & \cdots \\
\vdots & \vdots & \vdots & \ddots
\end{array}\right]
$$

the effect of the repeated coupling-uncoupling on the nominal system $\mathcal{T}_{0.5} \mathcal{T}^{T} \widetilde{\mathbf{K}}_{h} \mathcal{T}_{0.5}^{T}$ would amount to just averaging of matrix entries on the interstage boundaries having negligible effect on perturbed system global dynamic. Furthermore, its LU factors may be reused for different misalignment realizations. In absence of any perturbation the repeated coupling-uncoupling does not modify the nominal system

$$
\mathcal{T}^{T} \mathcal{T}_{0.5}=\boldsymbol{I}
$$

The combined results of two preceding sections for reduced order unbalance response analysis are summarized in Algorithm 1. The dynamic response analysis can be implemented in similar fashion.

\section{$3 \quad$ Numerical examples}

In this study we consider a rotor assembly consisting of four high pressure compressor integrally bladed discs composed of $36,60,84$ and 96 sectors respectively connected to a turbine disk featuring 120 sectors. The finite-element mesh of elementary sectors is depicted in Fig. 2. The assembly is analyzed in body attached frame that rotates about the undeformed centerline of the bearings with a constant speed. It is simply supported at the extremities, isotropic stiffness and damping at discrete nodal locations are taken into account. The bearing stiffness and damping coefficients are $k_{x x}=k_{y y}=4.58 \times 10^{8} \mathrm{~N} / \mathrm{m}$, $k_{x y}=k_{y x}=7.63 \times 10^{7} \mathrm{~N} / \mathrm{m}, c_{x x}=c_{y y}=1.52 \times 10^{6} \mathrm{Ns} / \mathrm{m}$ correspondingly, while internal rotor material damping is neglected.

Lowest frequency complex eigenmodes are calculated at 60 discrete frequency points $10 \mathrm{~Hz} \leq \Omega \leq 600 \mathrm{~Hz}$ with a step $10 \mathrm{~Hz}$ using multi-stage cyclic symmetry approach. The evolution of complex natural frequencies in rotating frame is given in Fig. 3(a). Same frequencies in inertial frame are depicted in Fig. 3(b), where the relationship between the frames is defined as $\omega_{\text {rotating }}=\omega_{\text {fixed }} \pm h \Omega$ with $h$ denoting the nodal diameter with 

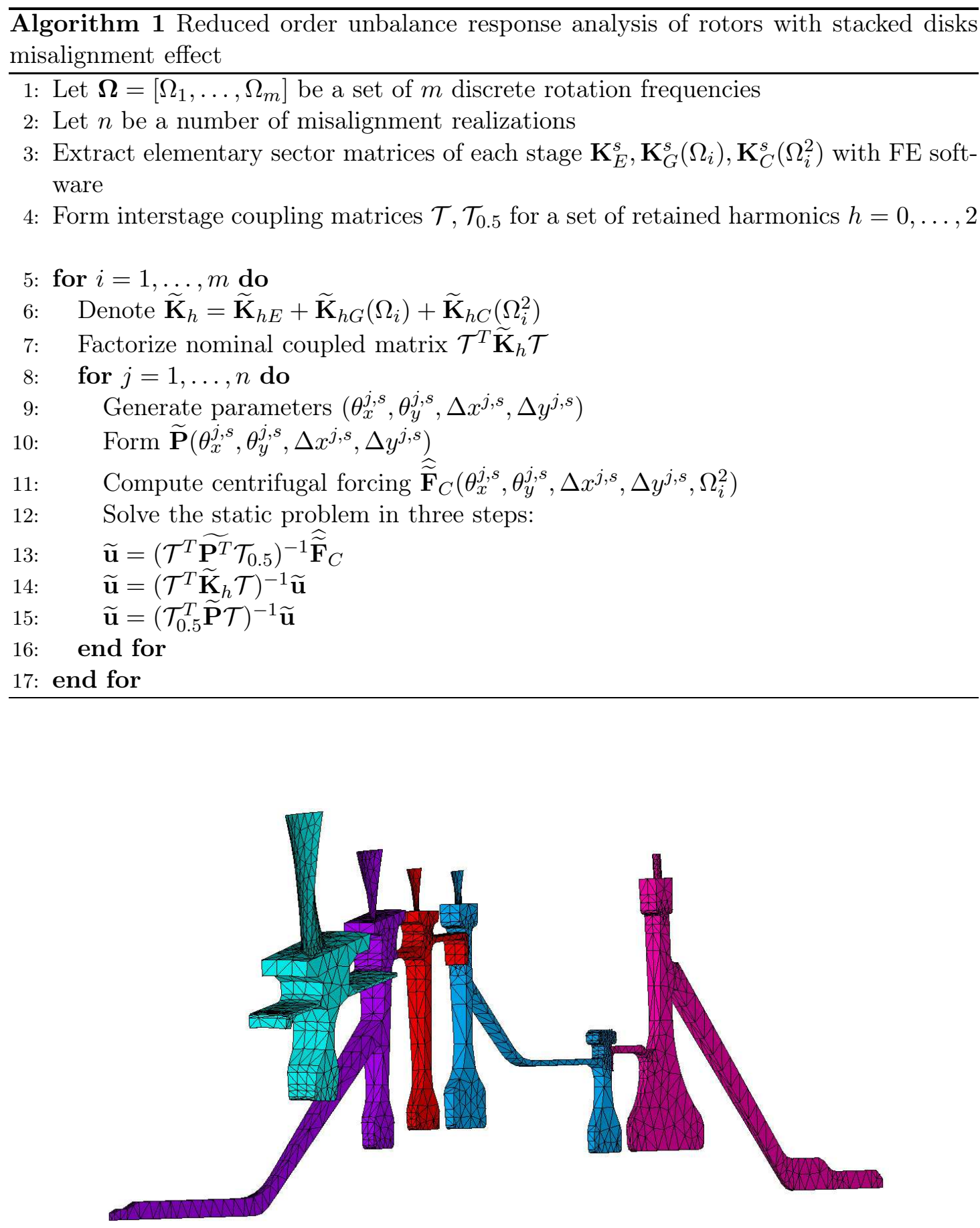

Figure 2: Finite element model of a multi-stage rotor assembly. 


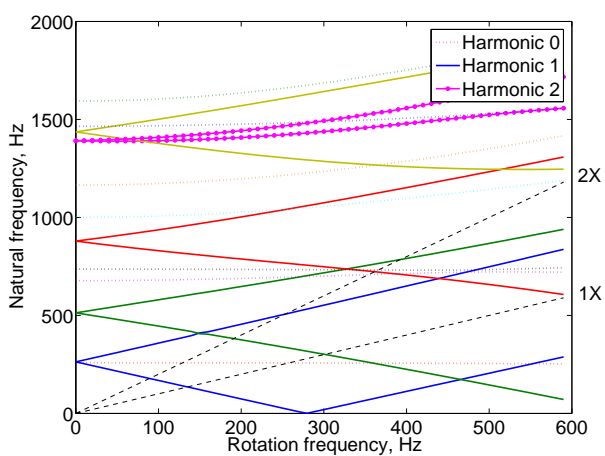

(a)

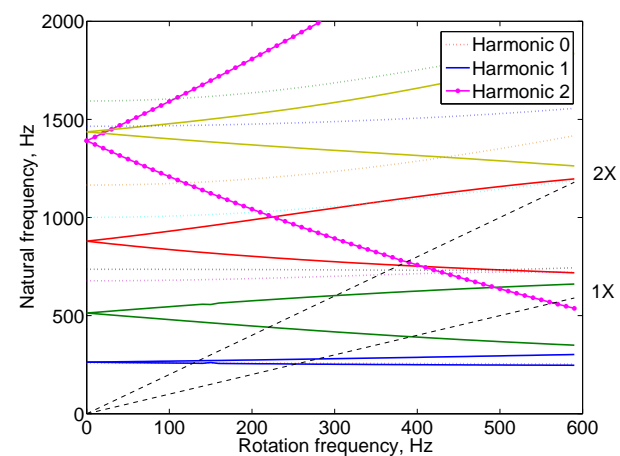

(b)

Figure 3: Evolution of natural frequencies of the nominal system with rotating speed in rotating frame (a), transformed to inertial frame (b). Synchronous whirl is marked as dashed line.

sign depending on the traveling wave direction. Note that synchronous and $2 \mathrm{X}$ whirls plotted to locate critical speeds are marked with dashed lines. The first critical speed in rotating frame occurs at about $\Omega=130 \mathrm{~Hz}$ corresponding to a forward whirl global bending mode. However, the first critical speed in inertial frame is observed at about $\Omega=280 \mathrm{~Hz}$, which corresponds to the cancelation of the apparent static stiffness in the rotating frame [5].

\subsection{Effect of misalignment on eigenmodes and system response}

First we investigate the effects of stacked disks misalignment on eigenmodes of the system. A misalignment scenario was introduced through a set of tilt angles and offsets presented in Tab. 1 applied to a full FE reference model and a set of lowest frequency complex eigenmodes was computed.

Table 1: Misalignment parameters

\begin{tabular}{|r|r|r|r|r|}
\hline Stage number & $\theta_{x},{ }^{\circ}$ & \multicolumn{1}{|c|}{$\theta_{y},{ }^{\circ}$} & $\Delta x, m m$ & $\Delta y, m m$ \\
\hline 1 & 0.01 & 0.015 & 1 & -0.95 \\
2 & -0.05 & -0.09 & 0.3 & 0.05 \\
3 & -0.06 & -0.045 & -0.75 & -2.8 \\
4 & 0.0443 & 0.0225 & 0.85 & 0.57 \\
5 & 0.08 & -0.095 & -0.39 & -0.45 \\
\hline
\end{tabular}

Figs. 4(a) and 4(b) present the difference between nominal and perturbed imaginary 
and real parts of complex eigenvalues calculated at $\Omega=200 \mathrm{~Hz}$. It should be noted that eigenvalues are not significantly affected by the misalignment, with maximum difference less than 0.3 percent for the lowest frequency mode. The observation strongly correlates with experimental data indicating that the resonance peaks at critical speeds can be reliably predicted by the Campbell diagram built from a nominal model. Eigenvec-

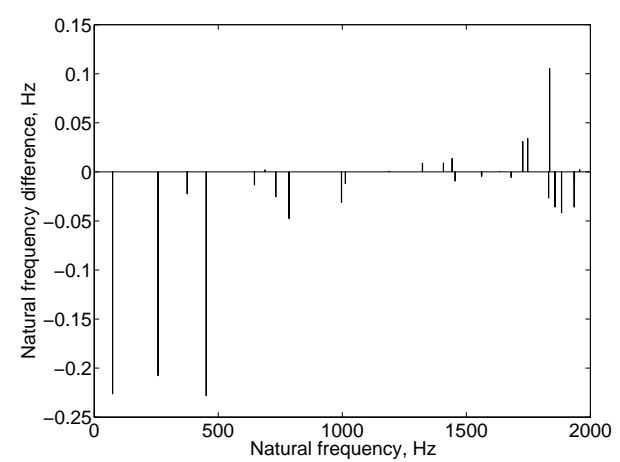

(a)

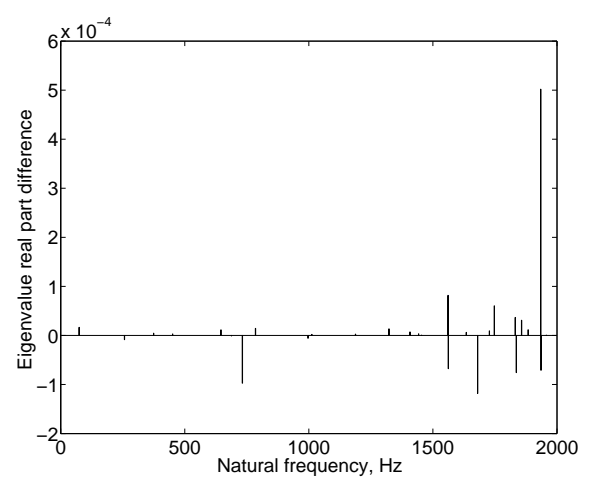

(b)

Figure 4: Difference between nominal and perturbed imaginary (a) and real (b) parts of complex eigenvalues calculated at $\Omega=200 \mathrm{~Hz}$.

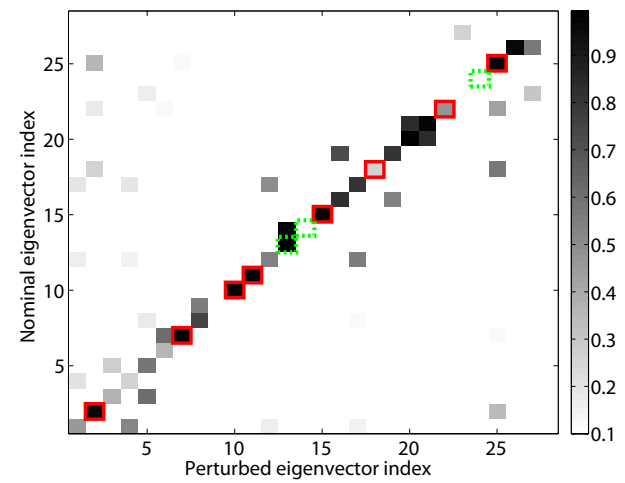

Figure 5: MAC value between nominal and perturbed complex eigenvectors calculated at $\Omega=200 \mathrm{~Hz}$. Harmonic 0 and 2 modes are highlighted with red solid and green dashed boxes respectively.

tors of the nominal and perturbed systems are compared in Fig. 5 in terms of complex MAC values. It appears that the eigenspace is affected more seriously by the effect of misalignment as reflected by low MAC values. Clearly, the mode distortion is the major reason why nominal eigenvectors cannot be used in modal projection based model reduction technique. It can also be seen in Fig. 5 that in general, harmonic one modes seem to be less stable under perturbation featuring lower MAC coefficients, whereas the 
modes of zero and second harmonic are strongly correlated with their original unperturbed counterparts. In order to gain better understanding of the effect of perturbation

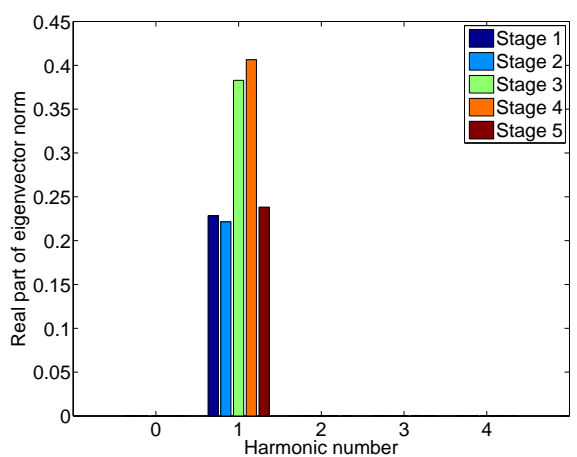

(a)

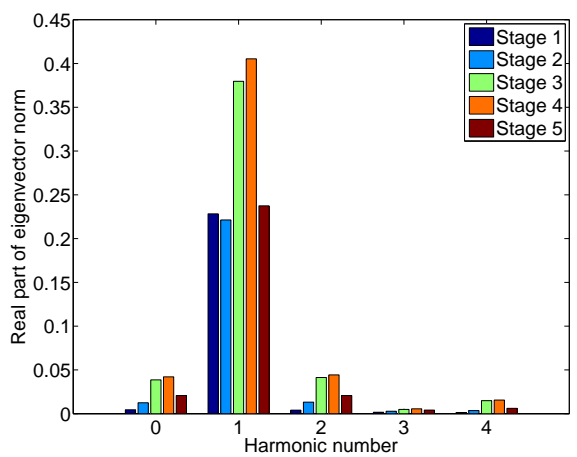

(c)

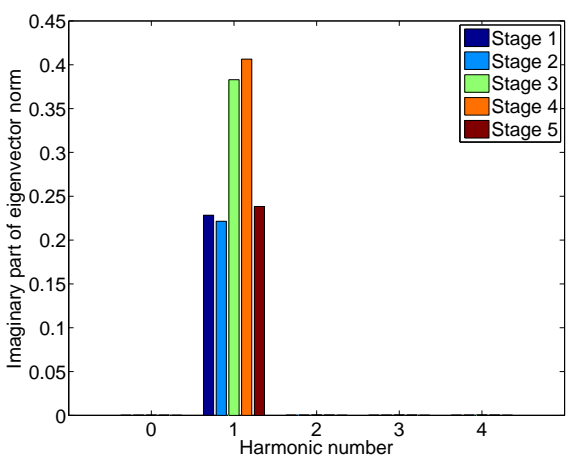

(b)

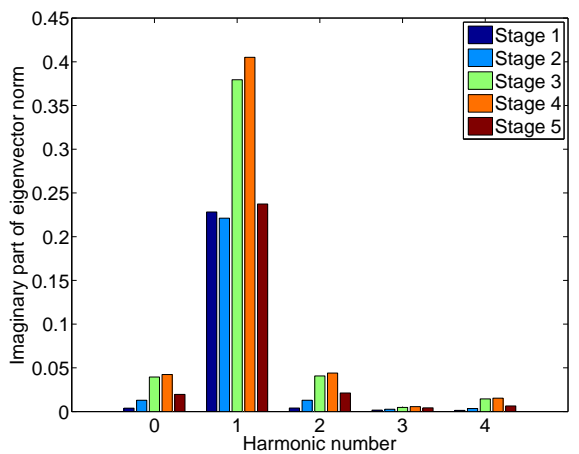

(d)

Figure 6: Harmonic content of the first bending mode corresponding to $74 \mathrm{~Hz}$ natural frequency at $\Omega=200 \mathrm{~Hz}$ and expressed in terms of norms of each individual stage. Norm of real part of nominal eigenvector (a), imaginary part (b), norm of real part of perturbed eigenvector $(\mathrm{c})$ and imaginary part(d).

on eigenvectors we calculate the harmonic content of real and imaginary parts of the first bending and the next in spectrum zero nodal diameter modes at $\Omega=200 \mathrm{~Hz}$, depicted in Figs. 6 and 7 correspondingly. Examination of the harmonic content of the modes yields significant insight. Both perturbed harmonic zero and harmonic one modes become contaminated mainly by the closest neighboring harmonics, and display other harmonic components to a lesser extent. The observation is consistent with the banded non-zero structure of the perturbed system matrices in Fourier domain - the amount of harmonic contamination is proportional to the norm of harmonic coupling blocks introduced by perturbation. The acquired additional harmonic content is most discernible in case of $257 \mathrm{~Hz}$ perturbed harmonic zero mode. The norm of harmonic one content in the imaginary part of eigenvector is higher than the one of the original harmonic zero 


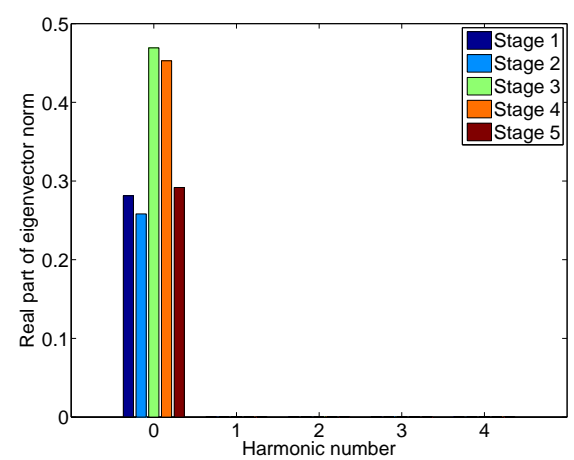

(a)

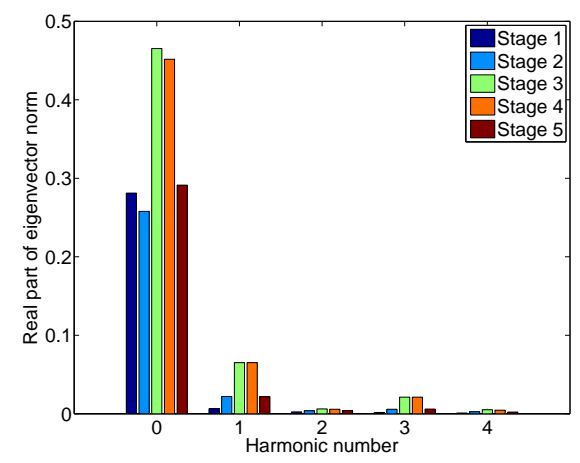

(c)

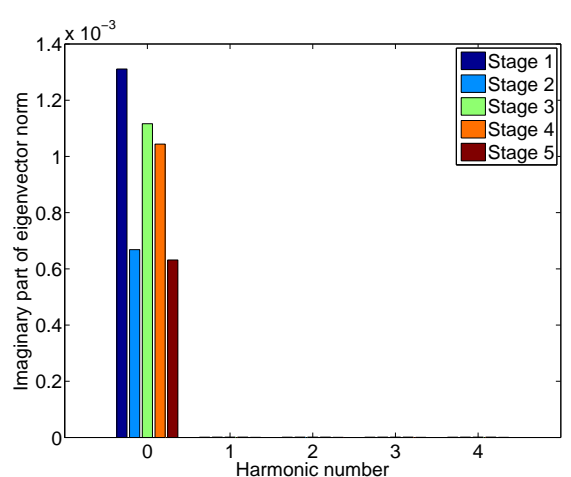

(b)

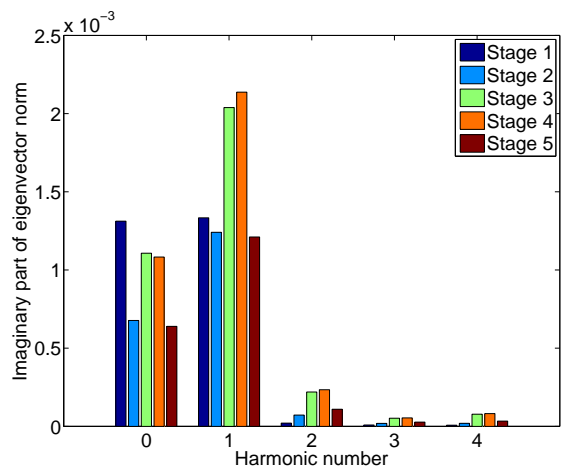

(d)

Figure 7: Harmonic content of zero nodal diameter mode corresponding to $257 \mathrm{~Hz}$ natural frequency at $\Omega=200 \mathrm{~Hz}$ and expressed in terms of norms of each individual stage. Norm of real part of nominal eigenvector (a), imaginary part (b), norm of real part of perturbed eigenvector $(\mathrm{c})$ and imaginary part(d).

content (see Fig. 7(d)), which can also be visualized using FE model in Fig. 8.

Next, we examine the effects of disk misalignment on static response. The response to centrifugal forcing for both nominal and misaligned system calculated at $\Omega=200 \mathrm{~Hz}$ is depicted in Fig. 9. While the nominal response is a pure harmonic zero displacement field, one can notice the dominance of harmonic one component in the response of the perturbed system. Fig. 10 shows the contribution of first four harmonics to the misaligned system unbalance response in $0-600 \mathrm{~Hz}$ frequency range. The harmonic decomposition is consistent with [22]. General perception is that both $1 \mathrm{X}$ and $2 \mathrm{X}$ (two times the rotation speed) components should be present in the response of a misaligned system with $1 \mathrm{X}$ being dominant, whereas the contribution of $2 \mathrm{X}$ vibration grows with severity of misalignment. The physical source of these effects is identified as a rotor bow and rotor asymmetry, respectively. Clearly, the magnitude of the response will be affected by the mode distortion phenomena discussed earlier that characterize misalignment. Thus, $257 \mathrm{~Hz}$ harmonic zero mode distorted by harmonic one component may be excited by 


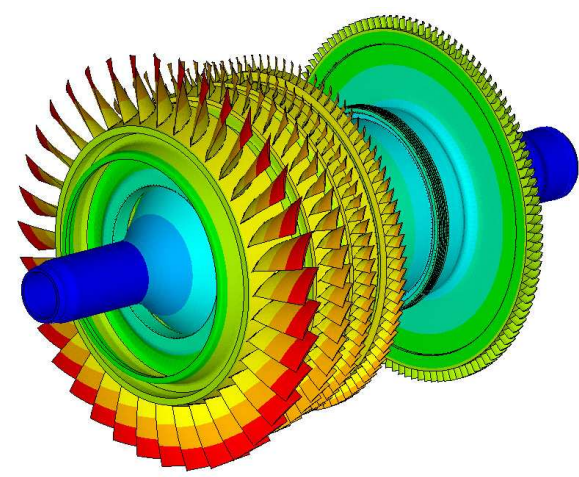

(a)

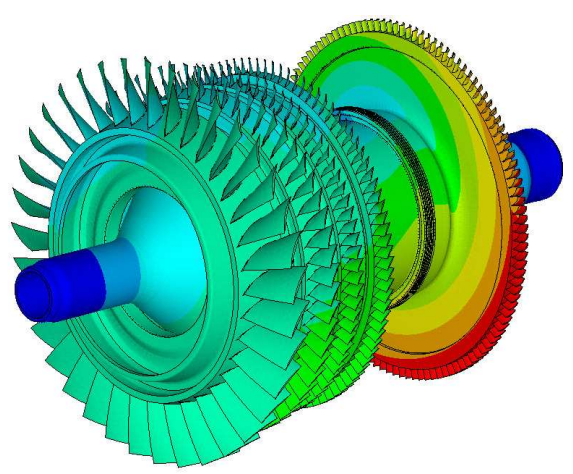

(b)

Figure 8: Perturbed harmonic zero modeshape corresponding to $257 \mathrm{~Hz}$ natural frequency at $\Omega=200 \mathrm{~Hz}$ rotational speed: real part (a) and imaginary part (b). The imaginary part of the modeshape is dominated by harmonic one component showing the effect misalignment.

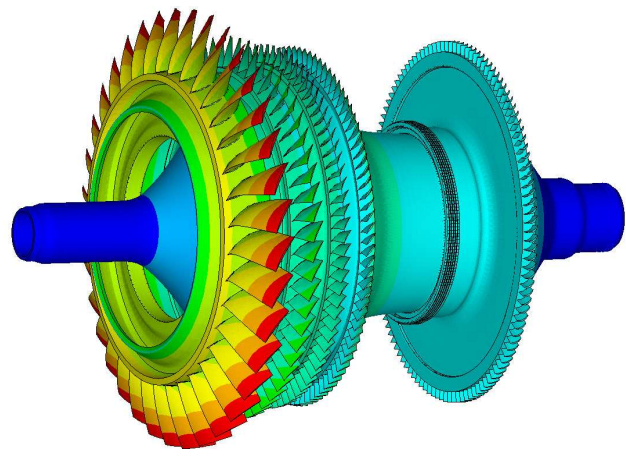

(a)

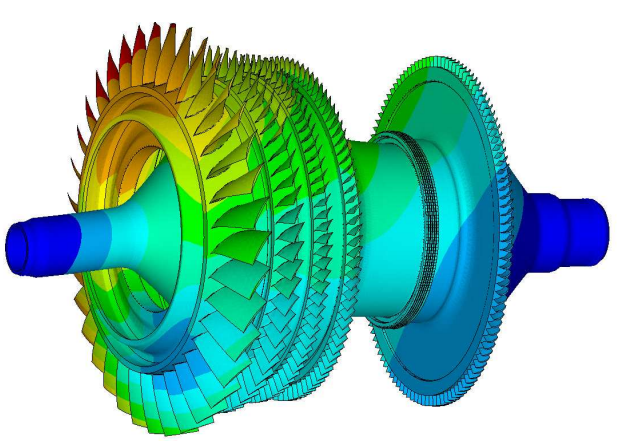

(b)

Figure 9: Nominal (a) and misaligned (b) system response under centrifugal forcing.

misaligned system centrifugal forcing dominated by both engine orders, zero and one, contributing significantly to $1 \mathrm{X}$ response magnification.

The effect of disk misalignment on harmonic response of the system excited by EO1 and EO2 forward traveling wave applied to bearing support nodes is shown in Fig. 11. It can be seen that the coupling between harmonic blocks introduced by perturbation, the reason of harmonic contamination of mode shapes, can cause significant response amplification, additional resonance peaks not observable in the nominal response as well as extra harmonic content other than the one of excitation. Observe that the misaligned system response to EO1 excitation is dominated by harmonic 0 component, whereas EO2 forcing brings about significant harmonic 1 response. 


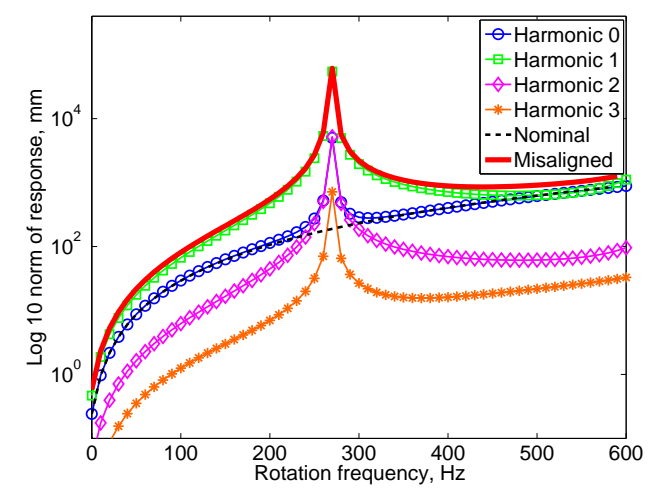

Figure 10: Comparison of nominal and misaligned systems unbalance response, the latter is shown decomposed into four harmonic components.

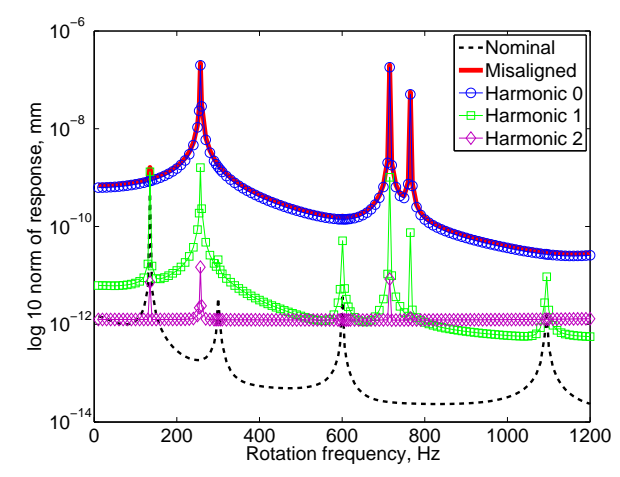

(a)

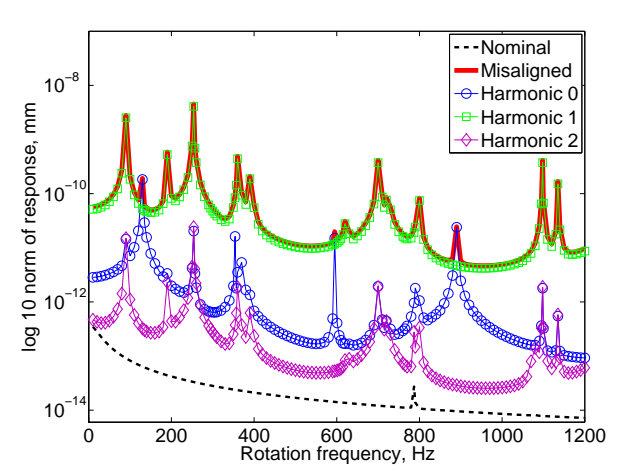

(b)

Figure 11: Comparison of nominal and misaligned system dynamic response under synchronous harmonic 1 (a) and $2 \mathrm{X}$ harmonic 2 (b) forward traveling wave excitation. The misaligned system response is shown along with its dominant harmonic components.

\subsection{Accuracy of the proposed method}

In the following example the proposed reduction technique is applied to form a reduced order model. To show its effectiveness the unbalance response is compared against the results calculated with full $\left(360^{\circ}\right)$ misaligned rotor-bearing system. Fig. 12(a) shows ROM accuracy in terms of norm of global response, same results are compared in Fig. 12(b) in terms of MAC correlation coefficients. Obviously, the ROM has been shown to accurately represent the centrifugal effects over the entire range of operating speeds in both suband super-critical regions. It slightly over-predicts the magnitude of response compared to the reference model. The MAC value is consistently over 0.97 showing the effect of deteriorating accuracy as rotation speed increases due to geometrical stiffness approximation. The response of nominal system excited by same unbalance forces is presented 


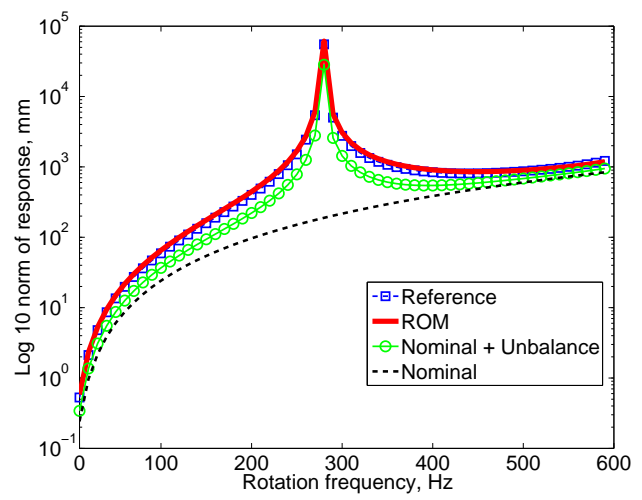

(a)

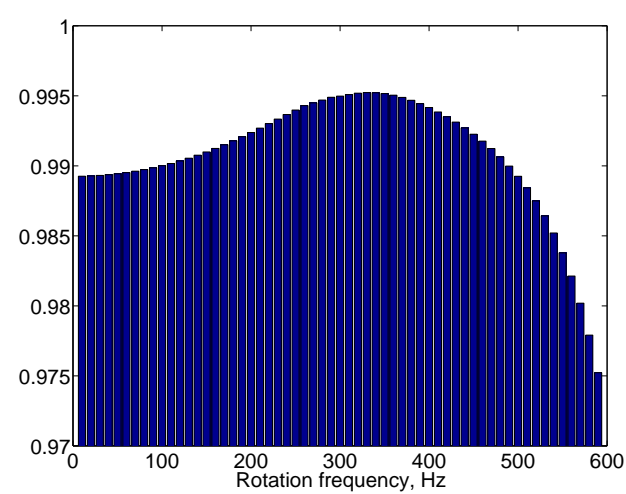

(b)

Figure 12: Norm of the unbalance response calculated with ROM, full $\left(360^{\circ}\right) \mathrm{FE}$ and unperturbed model excited by the unbalance forcing (a). Note that the latter consistently underestimates the response. MAC values of the unbalance response between ROM and reference FE model (b).

for comparison. It is evident that modeling of misalignment only with equivalent forces consistently underestimates the global response due to unmodeled effect of harmonic coupling and equivalently modal distortion.

\subsection{Statistical analysis example}

In this example, we consider a baseline model of the multi-stage rotor assembly introduced above (see Fig. 2). The reduced order model is constructed by projection, retaining first three Fourier harmonics, which results in a ROM of order 56,820 DOF. Owing to the fact that the most significant cause of excessive rotor vibration is rotor mass unbalance, which manifests itself as severe $1 \mathrm{X}$ vibration, the dynamic characteristic of primary interest is steady rotation speed unbalance response. Therefore, in Monte-Carlo simulations we carry out a static analysis under centrifugal loading measuring the deflection at bearing nodes. For simplicity, the nominal model is first perturbed by a set of misalignment parameters $\theta_{x}^{s}, \theta_{y}^{s}, \Delta x^{s}, \Delta y^{s}$ generated as statistically independent Gaussian random variables with zero mean and a standard deviation $\left(0.1^{\circ}, 0.1 \mathrm{~mm}\right)$. The random realizations of the amplitude of unbalance response at both bearing locations are shown in Fig. 13 along with the ensemble mean and percentiles. Observe, that the unbalance response levels at $99^{\text {th }}$ percentile can reach from 7 , between critical speeds, up to 20 , at a critical speed, times of those at $5^{\text {th }}$ percentile.

Next, MCS with a sample size 2000 is carried out to test the convergence of the response statistics. Fig. 14 displays ensemble mean and variance with respect to the number of samples; a sample size 1000 is found to be adequate for accurate analysis. Fig. 15 shows the pdf obtained from 1000 Monte-Carlo runs for three selected rotation frequencies, namely in the sub-critical region, at first critical speed and in the area close 


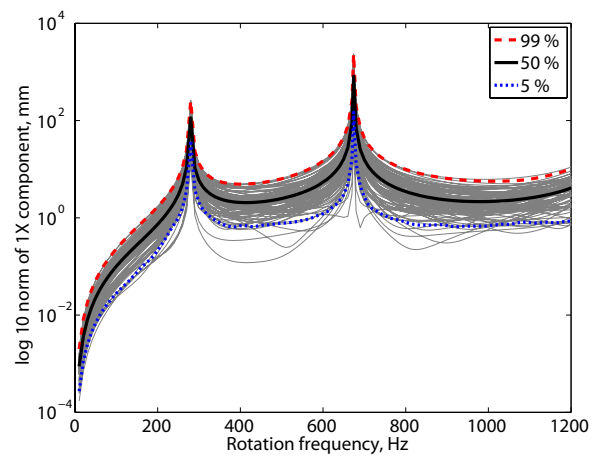

(a)

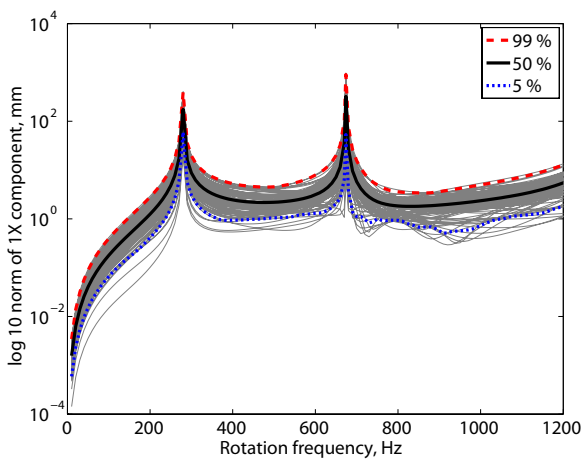

(b)

Figure 13: Direct Monte-Carlo simulation of the unbalance response with random misalignment parameters generated as statistically independent zero mean, $\left(0.1^{\circ}, 0.1 \mathrm{~mm}\right)$ standard deviation Gaussian random variables. Norm of $1 \mathrm{X}$ harmonic content of the unbalance response for 100 realizations, $99 \%, 50 \%$ and $5 \%$ of points at bearings 1 and 2 are shown in (a) and (b) correspondingly.

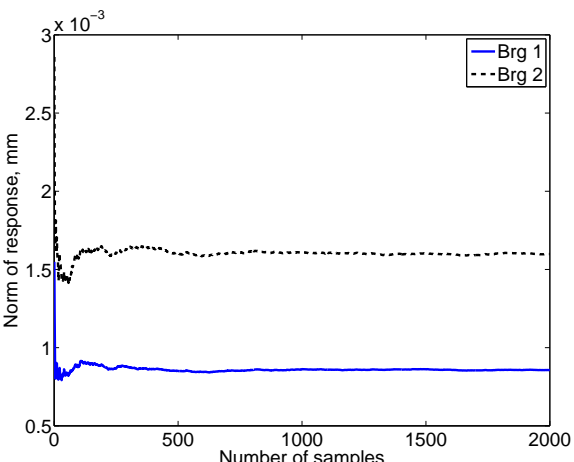

(a)

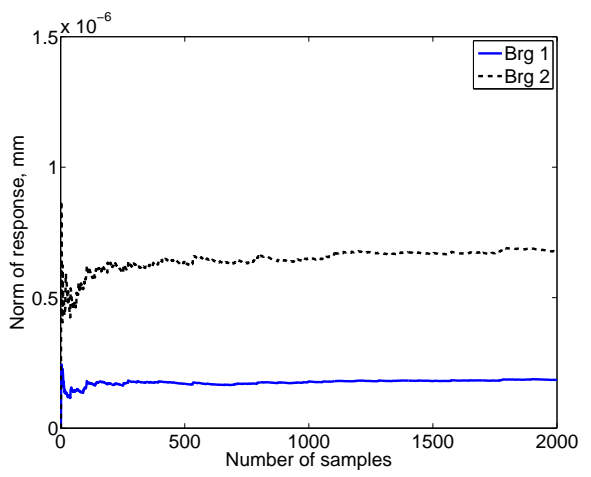

(b)

Figure 14: Evolution of the population mean (a) and variance (b) with the number of samples. Each iteration we calculate norm of $1 \mathrm{X}$ content of the unbalance response at two bearings at $\Omega=10 \mathrm{~Hz}$.

to the second critical speed. The observable differences in pdf shapes of two bearings at three rotation speeds are consistent with the corresponding bending modeshapes: larger variation is expected for a modeshape dominated by motion of that part of structure.

The statistically quantified levels of unbalance response are of importance while selecting robust designs and manufacturing tolerances to avoid large amplitude response within the operating range. An important ramification of imbalance induced excessive $1 \mathrm{X}$ vibration, the passage through a critical speed, is illustrated in the following example. The $99^{t h}$ percentile of the unbalance response norm is computed at first critical speed 


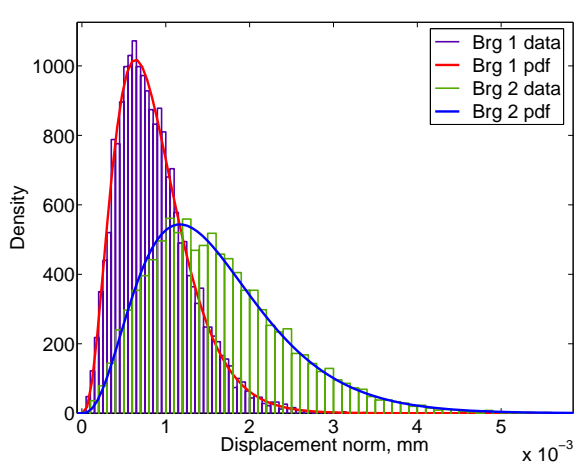

(a)

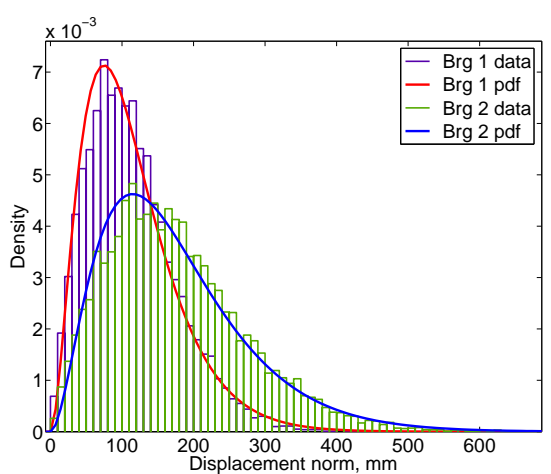

(b)

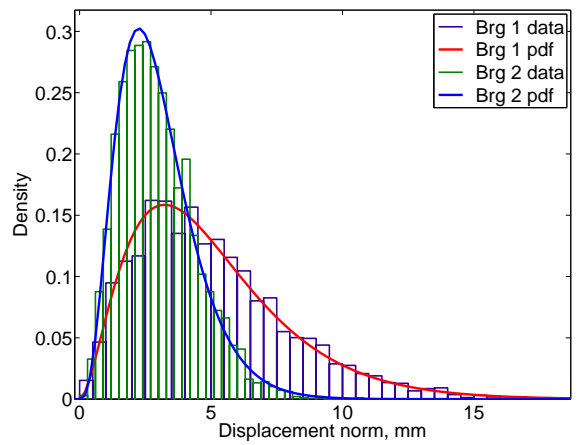

(c)

Figure 15: Probability density functions of the static response at two bearings (1X component) obtained at $\Omega=10 \mathrm{~Hz}$ (a), $\Omega=280 \mathrm{~Hz}$ (b) and $\Omega=590 \mathrm{~Hz}$ (c). Note larger variation in response at second bearing in the subcritical region and at first critical speed. As we approach the second critical speed, the distribution at first bearing grows wider consistent with the first and second bending modeshapes.

$\Omega=280 \mathrm{~Hz}$ with two standard deviation values of misalignment parameters $\left(0.5^{\circ}, 0.5\right.$ $\mathrm{mm})$ and $\left(1^{\circ}, 1 \mathrm{~mm}\right)$ for each stage separately, while those of other disks are kept at $\left(0.1^{\circ}, 0.1 \mathrm{~mm}\right)$ level. Assuming a typical industrial situation where an optimized stacking orientation for each stage of the rotor assembly is a function of all individual disks random geometries, we introduce a simple decreasing statistical dependence between misalignment parameters $\theta_{x}^{s}, \theta_{y}^{s}, \Delta x^{s}, \Delta y^{s}$ of different stages $s=1, \ldots 5$. Thus, the selected correlation coefficients are $\rho_{s, s \pm 1}=0.9, \rho_{s, s \pm 2}=0.7, \rho_{s, s \pm 3}=0.4$ and $\rho_{s, s \pm 4}=0.1$. Fig. 16 shows the influence of the amplitude or random geometry variation of each individual stage on the variation of global response calculated for two bearings at first critical speed. The observed changes in $99^{\text {th }}$ percentile of the response level with the additional uncertainty at one stage agree with the physical intuition. It can be observed that the relative importance of the manufacturing uncertainties in the geometry of the first and the last interfaces outweighs the ones of the middle stages suggesting tighter tolerances 


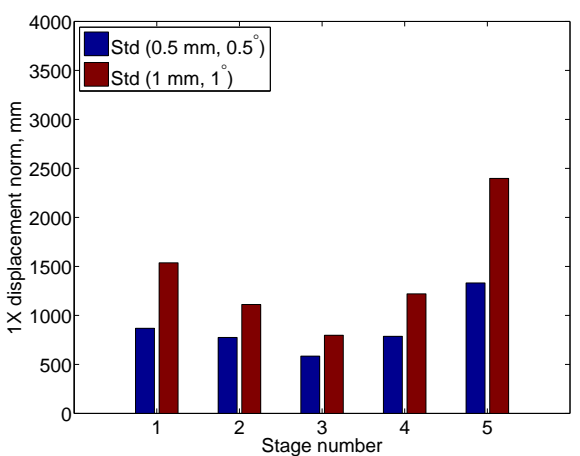

(a)

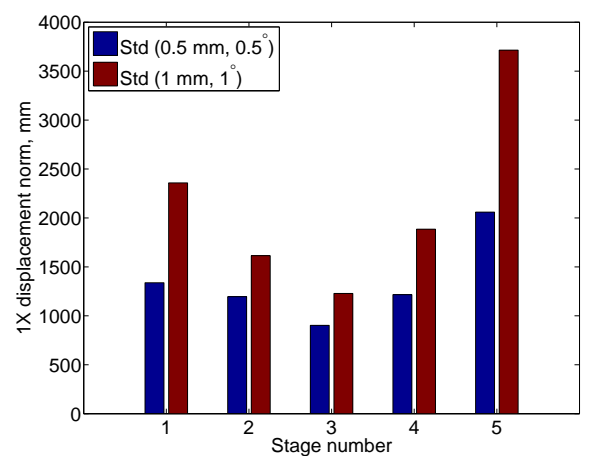

(b)

Figure 16: $99^{\text {th }}$ percentile of the unbalance response norm calculated at $\Omega=280 \mathrm{~Hz}$ resonance frequency at bearing 1 (a) and bearing 2 (b) obtained by increasing standard deviation of random input parameters to $\left(0.5^{\circ}, 0.5 \mathrm{~mm}\right)$ and $\left(1^{\circ}, 1 \mathrm{~mm}\right)$ for each stage separately, while those of others are kept at $\left(0.1^{\circ}, 0.1 \mathrm{~mm}\right)$.

to ensure a reliable performance.

All the numerical experiments were conducted on an Intel Xeon Quad-Core $2.66 \mathrm{GHz}$ workstation, coded in FORTRAN employing PARDISO direct sparse solver. For comparison, one iteration of MCS with the ROM featuring 56,820 DOF requires $0.5 \mathrm{G}$ of RAM taking approximately $5 \mathrm{~s}$ counting both reduced order model update and static analysis. An equivalent accuracy full order model has 929,160 DOF requiring 4 min for static analysis and $17 \mathrm{G}$ of memory (in-core version), whereas a realistic introduction of disk misalignment into the full model would require $\mathrm{FE}$ reanalysis.

\section{Concluding remarks}

In this paper we have introduced a new parameterized model order reduction technique for vibration analysis of rotor assemblies with variations in geometry induced by disk misalignment using 3D FE formulation. The reduced model has been obtained by orthogonal projection of system equations onto a subspace spanned by a few Fourier basis vectors corresponding to first three harmonics. The disks misalignment has been introduced as perturbation to system matrices as well as corresponding centrifugal forces. It has been shown that the Fourier subspace can be efficiently reused to project the misalignment geometrical perturbation terms owing to their special structure. The latter can be generated on-line as multiplicative perturbation, a function of small number of measurable eccentricity and tilt angle parameters, without resorting to FE software. Computational strategies have been provided that significantly simplify and accelerate repeated ROM update and solution using modern direct sparse solver technology. Of particular interest is the ability to repetitively introduce variation in geometry where the modal projection based methods usually fail or numerically ineffective. The algorithm can therefore be 
advantageous in non-intrusive direct uncertainty quantification methods at design stage and on-line optimum stacking at rotor assembly stage, specifically for modern light flexible rotor designs operating in supercritical regions. The accuracy and performance of the algorithm implementation has been illustrated with representative simulation examples, the results have been shown to match reference system over a practical range of geometrical parameter variations and rotational speeds. The development has also been instrumental in understanding of the inaccuracy of traditional analysis methods. It has been shown numerically that the non-isotropic stiffness variation introduced as sparse blocks coupling neighboring harmonics is the origin of additional $1 \mathrm{X}$ and $2 \mathrm{X}$ content in the response of misaligned system. Continuing research effort is still needed with respect to performance and accuracy of the proposed method and for a more general and effective methodology accommodating other types of deviations from nominal symmetry over a larger range of applications.

\section{References}

[1] N. Bachschmid, P. Pennacchi, and A. Vania. Identification of multiple faults in rotor systems. Journal of Sound and Vibration, 254(2):327-366, 2000. doi:10.1006/jsvi.2001.4116.

[2] M. Castanier and C. Pierre. Modeling and analysis of mistuned bladed disk vibration: Status and emerging directions. Journal of Propulsion and Power, 22(2): 2285-2298, 2006.

[3] E. Chatelet, D. Lornage, and G. Jacquet-Richardet. A three dimensional modeling of the dynamic behavior of composite rotors. International Journal of Rotating Machinery, 8(3):185-192, 2002. doi:10.1155/S1023621X02000179.

[4] E. Chatelet, F. D'Ambrosio, and G. Jacquet-Richardet. Toward global modelling approaches for dynamic analyses of rotating assemblies of turbomachines. Journal of Sound and Vibration, 282:163-178, 2005.

[5] D. Combescure and A. Lazarus. Refined finite element modeling for the vibration analysis of large rotating machines: Application to the gas turbine modular helium reactor power conversion unit. Journal of Sound and Vibration, 318:1262-1280, 2002. doi:10.1016/j.jsv.2008.04.025.

[6] A. Das and J. Dat. Reduced model of a rotor-shaft system using modified serep. Mechanics Research Communications, 35:398-407, 2008. doi:10.1016/j.mechrescom.2008.04.004.

[7] S. Edwards, A. Lees, and M. Friswell. Experimental identification of excitation and support parameters of a flexible rotor-bearing-foundation system from a single run-down. Journal of Sound and Vibration, 235(5):963-992, 2000. doi:10.1006/jsvi.1999.2779. 
[8] M. Friswell and D. Inman. Reduced-order models of structures with viscoelastic components. AIAA Journal, 37(10):1318-1325, 1999. doi:10.2514/2.603.

[9] M. Friswell, J. Penny, and S. Garvey. Model reduction for structures with damping and gyroscopic effects. In Proceedings of ISMA-25, Leuven, Belgium, September 2000 .

[10] V. Ganine, M. Legrand, H. Michalska, and C. Pierre. A sparse preconditioned iterative method for vibration analysis of geometrically mistuned bladed disks. Computers and Structures, 87:342-354, 2009. doi:10.1016/j.compstruc.2008.12.011. URL http://hal . archives-ouvertes.fr/hal-00363296/.

[11] G. Genta. Dynamics of Rotating Systems. Springer, New York NY, 2005.

[12] G. Jacquet-Richardet, G. Ferraris, and P. Rieutord. Frequencies and modes of rotating flexible bladed disc-shaft assemblies: a global cyclic symmetry approach. Journal of Sound and Vibration, 191(5):901-915, 1996. doi:10.1016/j.jsv.2004.02.035.

[13] D. Kammer. Test-analysis-model development using exact model reduction. The International Journal of Analytical and Experimental Modal Analysis, 2(4):174-179, 1987.

[14] Y. Khulief and M. Mohiuddin. On the dynamic analysis of rotors using modal reduction. Finite Elements in Analysis and Design, 26(1):41-55, 1997. doi:10.1016/S0168-874X(96)00070-4.

[15] D. Laxalde, J.-P. Lombard, and F. Thouverez. Dynamics of multistage bladed disks systems. Journal of Engineering for Gas Turbines and Power, 129(4):1058-1064, 2007. doi:10.1115/1.2747641. URL http://hal . archives-ouvertes.fr/hal-00363296/.

[16] L. G. Maqueda, O. Bauchau, and A. Shabana. Effect of centrifugal forces on the finite element eigenvalue solution of a rotating blade: a comparative study. Multibody System Dynamics, 19:281-302, 2008. doi:10.1007/s11044-007-9070-6.

[17] R. Markert, R. Platz, and M. Seidler. Model based fault identification in rotor systems by least squares fitting. International Journal of Rotating Machinery, 7(5): 311-321, 2001. doi:10.1155/S1023621X01000264.

[18] P. Pennacchi and A. Vania. Identification of a generator fault by model-based diagnostic techniques. International Journal of Rotating Machinery, 10(4):293-300, 2004. doi:10.1155/S1023621X04000302.

[19] P. Pennacchi, N. Bachschmid, A. Vania, G. Zanetta, and L. Gregori. Use of modal representation for the supporting structure in model-based fault identification of large rotating machinery. Mechanical Systems and Signal Processing, 20:662-681, 2006. doi:10.1016/j.ymssp.2004.11.006. 
[20] J. Sawicki and W. Gawronski. Balanced model reduction and control of rotor-bearing systems. Journal of Engineering for Gas Turbines and Power, 119:456-463, 1997. doi:10.1115/1.2815596.

[21] J. Shortle and M. Mendel. Predicting dynamic imbalance in rotors. Probabilistic Engineering Mechanics, 11:31-35, 1996. doi:10.1016/0266-8920(95)00025-9.

[22] J. Sinha, A. Lees, and M. Friswell. Estimating unbalance and misalignment of a flexible rotating machine from a single run-down. Journal of Sound and Vibration, 272(3-5):967-989, 2004. doi:10.1016/j.jsv.2003.03.006 .

[23] R. Stephenson and K. Rouch. Modeling rotaing shafts using axisymmetric solid finite elements with matrix reduction. Journal of Vibration and Acoustics, 115:484-489, 1993. doi:10.1115/1.2930376.

[24] D. Sundararajan. The Discrete Fourier Transform. Theory, Algorithms and Applications. World Scientific, Singapore, 2001.

[25] D. Thomas. Dynamics of rotationally periodic structures. International Journal for Numerical Methods in Engineering, 14(1):81-102, 1979. doi:10.1002/nme.1620140107.

\section{Appendix}

Consider a two dimensional signal

$$
\begin{gathered}
x[n, m]=\cos \left(\frac{2 \pi r n}{N}\right) \delta(|n-m|) \\
n, m=0, \ldots, N-1
\end{gathered}
$$

Its Fourier domain representation can be expressed as

$$
\begin{gathered}
x[u, v]=\sum_{m=0}^{N-1} \sum_{n=0}^{N-1} w_{N}^{-m v} w_{N}^{n u} \cos \left(\frac{2 \pi r n}{N}\right) \delta(|n-m|)= \\
=\sum_{m=0}^{N-1} w_{N}^{-m v} w_{N}^{m u} \cos \left(\frac{2 \pi r m}{N}\right)=\frac{1}{2} \sum_{m=0}^{N-1} w_{N}^{m(u-v-r)}+\frac{1}{2} \sum_{m=0}^{N-1} w_{N}^{-m(v-u-r)}= \\
=\frac{1}{2} \delta((u-v)-r)_{\bmod N}+\frac{1}{2} \delta((v-u)-(N-r))_{\bmod N}= \\
=\frac{1}{2} \delta((u-v)-r)+\frac{1}{2} \delta((u-v)-(N-r))+\frac{1}{2} \delta((v-u)-r)+\frac{1}{2} \delta((v-u)-(N-r)) \\
u, v=0, \ldots, N-1
\end{gathered}
$$

where $w_{N}^{-m v}$ denotes complex exponential $e^{-\mathrm{j} \frac{2 \pi}{N} m v}, \delta$ is the Dirac delta function. 